\title{
Aktuelles zum MTA-Reformgesetz
}

Das BMG überarbeitet das MTA-Gesetz. Ärztliche Berufsverbände und Gesellschaften, so auch die DRG und der BDR, haben Stellungnahmen abgegeben. Nachfolgend finden Sie die Stellungnahme des BDR an das Bundesministerium für Gesundheit zum MTA-Reform-Gesetz.

\section{Entwurf eines Gesetzes zur} Reform der technischen Assistenzberufe in der Medizin und zurÄnderung weiterer Gesetze (MTA-Reform-Gesetz)

Sehr geehrte Frau Redert, sehr geehrte Damen und Herren,

als Berufsverband der Deutschen Radiologen dürfen wir zu dem Referentenentwurf des MTA-Reform-Gesetzes nachfolgend Stellung nehmen und darum bitten, für zukünftige Korrespondenz im Verteiler berücksichtigt zu werden. Der BDR vertritt die Interessen der Radiologen in Niederlassung und Krankenhaus, die zum Betrieb ihrer Praxen und Abteilungen zwingend auf die Unterstützung durch MTA angewiesen sind.

Auch wenn MTA derzeit noch nicht als Mangelberuf eingestuft werden, besteht akuter Nachwuchsmangel und können schon jetzt offene Stellen mangels Bewerber*innen nicht mehr nachbesetzt werden.

Ziel der Gesetzesinitiative muss es deshalb auch sein, die Attraktivität des Berufes zu steigern und Möglichkeiten der Nach- bzw. Angleichungsqualifikation für andere medizinische Fachberufe zu schaffen (wie dies auch der DVTA in seiner Antwort vom 05.07.2019 an die Bund-Länder-Arbeitsgruppe zum Gesamtkonzept zur Neuord- nung und Stärkung der Ausbildung der Gesundheitsfachberufe fordert).

Im Einzelnen bitten wir um Berücksichtigung folgender Aspekte im weiteren Gesetzgebungsverfahren:

1. zu $\S 5$ Abs. 2 Nr. 1 (und entsprechend $\S 10$ Abs. 1 Nr. 1)

Wie in der bisherigen Fassung des $\S 9$ Abs. 1 Nr. 2a MTA-Gesetzes sieht auch $\S 5$ Abs. 2 Nr. 1 nunmehr vor, neben der radiologischen Bildgebung auch ,anderer bildgebender Verfahren“" als vorbehaltene Tätigkeiten aufzunehmen. Dies ist aus unserer Sicht für die Definition eines Vorbehaltskataloges zu unbestimmt. Die Aufzählung vorbehaltener Tätigkeiten muss aus sich heraus klar und eindeutig sein, zumal wenn Verstöße strafbewehrt sind. Der Zusatz „anderer bildgebender Verfahren“ muss deshalb ersatzlos entfallen.

Es besteht auch keine Notwendigkeit der Erstreckung auf weitere Methoden der Bildgebung, nachdem der Vorbehaltskatalog des MTA-Gesetzes wesentlich auch auf dem Aspekt des Strahlenschutzes bei Anwendung ionisierender Strahlung beruht und andere derzeit übliche Bildgebungsverfahren wie Sonographie oder MRT nicht mit einer vergleichbaren Gefährdung für die Patienten verbunden sind.

\section{2. zu $\oint 6$ Nr. 2}

Der Zusatz Heilpraktikerinnen und Heilpraktiker ist zu streichen. Es gibt dafür keinen denkbaren Anwendungsfall, nachdem keine der derzeit üblichen Ausbildungen oder Prüfungen für Heilpraktiker bildgebende Verfahren im Bereich der Medizin beinhalten. Auch in $\S 145 \mathrm{StrSchV}$, dem die Regelung des $\S 6$ strukturell entspricht, ist eine Tätigkeit durch Heilpraktiker nicht vorgesehen.

\section{3. zu $§ 6$ Nr. 8}

Uns ist bekannt, dass verschiedentlich die Streichung dieser Regelung gefordert wird. Sie muss indes unbedingt erhalten bleiben, da sie unmittelbar mit der Regelung des $\S 145$ Abs. 2 Nr. 5 StrSchV (2018) korreliert.

4. Das MTBG sollte dringend die Möglichkeiten eröffnen, Angehörigen anderer medizinischer Fachberufe, die während ihrer Ausbildung oder dauerhaften Berufsausübung zulässig vorbehaltene Tätigkeiten ausüben die Berufsqualifikation unter Anrechnung bereits erworbener Qualifikationen zu ermöglichen.

So sollte konkret § 15 Abs. 1 MTBG nicht nur die Anrechnung fachschulische oder hochschulische Ausbildung und damit nur theoretischen und ggf. praktischen Unterricht zu ersetzen, sondern auch langjährige praktische Erfahrung. So sollte z. B. die Tätigkeit i. S. d. § 6 Nr. 8 in einer radiologischen Praxis oder Krankenhausabteilung nach einer Dauer von 5 Jahren als Kenntniserwerb praktischer Ausbildung anrechenbar sein.

Darüber hinaus sollten ggf. die Anerkennungsmöglichkeiten des gesamten 4. Teils des Gesetzes nicht nur für die Anerkennung von Qualifikationen außerhalb des Geltungsbereichs des Gesetzes, sondern auch für die Anerkennung entsprechender Qualifikationen in Deutschland gelten. Teil 4 des Gesetzes schafft mit den $\S \S 41$ bis 52 ein abgewogenes System der Anerkennung von Berufsqualifikationen, die sicherstellen, dass die Qualität der Ausbildung gewährleistet bleibt.

5. Der Erfüllungsaufwand des Gesetzes wird für die Wirtschaft als „Sehr gering“ mit $€ 60000$,- p. a. bzw. $€ 20000$,- einmalig für den Abschluss von Kooperationsverträgen 
prognostiziert. Mehrkosten für die Vergütung von Auszubildenden sind für niedergelassene Ärzte als Träger der praktischen Ausbildung über die Vergütungssysteme des EBM und der GOÄ nicht abgebildet und in diesem Umfang nicht refinanzierbar.

Sowohl in den laufenden Verhandlungen zur Novellierung der GOÄ als auch bei der
Weiterentwicklung des EBM sind für die betroffenen Fachgruppen diese Kosten zu berücksichtigen. Alternativ besteht die Möglichkeit, zumindest im GKV-System entsprechende Ausbildungszuschüsse verpflichtend vorzusehen.

Ohne Kompensation des Mehraufwands auch in der dargestellten Höhe, ist die prak- tische Ausbildung durch niedergelassene Ärzte und Einrichtungen nicht darstellbar.

Für Rückfragen stehen wir gerne zur Verfügung,

mit freundlichen Grüßen Markus Henkel

Geschäftsführer 